\title{
Preparation and Dissolution of Solid Dispersions of Sanguis Draconis
}

\author{
XING Chang Min ${ }^{1, a}$, WANG Xue Ming ${ }^{1, b}$, CAO Kuan²,c ${ }^{2}$, LIU Hui Chao ${ }^{2, d}$, LIU \\ Yong $^{2, \mathrm{e}^{*}}$ and LI Nan ${ }^{1, f^{*}}$ \\ 'Department of Digestion, The $309^{\text {th }}$ Hospital of PLA, Beijing 100091, China \\ ${ }^{2}$ College of Mechanical and Electrical Engineering, Beijing University of Chemical Technology, \\ Beijing 100029, China

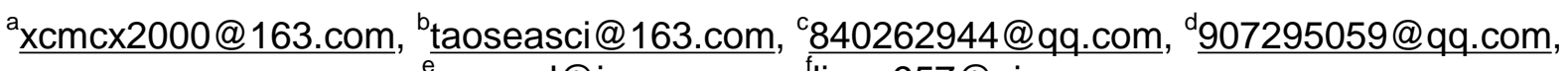 \\ 'yongsd@iccas.ac.cn, linan957@sina.com
}

Keywords: sanguis draconis, solid dispersions, dissolubility, polyethylene glycol, polyvinyl pyrrolidone

\begin{abstract}
Sanguis draconis is a precious traditional Chinese medicine, but it is not soluble in water, and it is difficult to use in type of injection. Sanguis draconis has a low bioavailability, because the coarse particles of draconis can be absorbed very little. In present research, solid dispersions of sanguis draconis were prepared using solvent method and melting methods with PEG-6000 and $\mathrm{PVP}_{\mathrm{K} 30}$ as carriers. The dissolubility in vitro was determined by UV method. Results showed that higher carrier ratio led to faster drug dissolution. The dissolubility of solid dispersions with $\mathrm{PVP}_{\mathrm{K} 30}$ was faster than that with PEG-6000. The solid dispersions of sanguis draconis can improve its dissolution obviously, which promotes the absorption and bioavailability of this medicine.
\end{abstract}

\section{Introduction}

Sanguis draconis is a precious traditional Chinese medicine. It has important functions such as removing blood stasis, relieving pain, hemostatic and promoting tissue regeneration. It was broadly used for the symptom of traumatic injury and internal stasis pain [1]. Pharmacological effects of sanguis draconis mainly showed in hemostasis and invigorating the circulation of blood, blood scattered silt, and effect on cardiovascular function [2]. Sanguis draconis is commonly used for blood scattered silt, and the treatment of wounds and skin sores diseases in traditional medicine. Sanguis draconis is used in the clinical treatment of a variety of refractory wounds because of a significant role in promoting blood circulation and myogenic, and it has a good effect. Sanguis draconis is resin type of medicine, mainly containing a variety of complex ingredients such as dracorhodin, dracorubin, other flavonoids and triterpenoids. Flavonoid is the main component using for invigorating the circulation of blood. Draconis is not soluble in water and is not easy to use in injection, so it is used for oral preparations, enemas, etc. Sanguis draconis has a low bioavailability because of the coarse particles of draconis which the body absorbs very little. As we all know, $\mathrm{PVP}_{\mathrm{k} 30}$ is a water-soluble polymer and has good biocompatibility, so it is widely used in the field of drugs, cosmetics, food, other medicine and life [3]. PEG-6000 is a non-toxic, non-irritating, slightly bitter taste. It has a good solubility in water and compatibility with many organic components. They have excellent lubricity, moisture, dispersion, bonding agents, antistatic agents and softeners. $\mathrm{PVP}_{\mathrm{k} 30}$ and PEG-6000 have a very wide range of applications in cosmetics, pharmaceutical, chemical fiber, rubber, plastics, paper, paint, electroplating, pesticides, metal processing, food processing and other industries. Therefore, in order to improve the dissolution and dissolution rate of draconis and the low bioavailability of draconis, taking into account that $\mathrm{PVP}_{\mathrm{k} 30}$ or PEG-6000 can be as a carrier, we can accelerate the dissolution and dissolution rate of sanguis draconis to improve the bioavailability of draconis by mixed the different carriers with sanguis draconis each other by solid dispersion technology. Sanguis draconis- $\mathrm{PVP}_{\mathrm{k} 30}$ was prepared by solvent method, using different proportions of draconis and $\mathrm{PVP}_{\mathrm{k} 30}$. Solid dispersion of sanguis draconis-PEG-6000 was prepared by Melting method in different proportions of sanguis draconis and PEG-6000. Dissolution test showed that dissolution of solid 
dispersion powder in all proportions was much higher than that of original powder medicament, and the dissolution rate of drug was increased with the increase of the amount of the carrier. The contrast experiments found that $\mathrm{PVP}_{\mathrm{k} 30}$ was better than PEG-6000 as a carrier in terms of the dissolution effect. We found that solid dispersion of sanguis draconis could accelerate its dissolution to expect to improve bioavailability.

\section{Experiment}

Solution Preparation Sanguis draconis- $\mathrm{PVP}_{\mathrm{k} 30}$ solid dispersion was prepared by solvent method [4-6]. Powder of sanguis draconis (Grand Kunming Pharmaceutical Factory) and $\mathrm{PVP}_{\mathrm{k} 30}$ (Beijing Yi li Fine Chemicals Co., Ltd) were weighed by the weight ratio after 60 mesh sieve filtration. An appropriate amount of anhydrous ethanol was added to dissolve the drug and the carrier. The solutions were placed in the evaporating dish, stirred to shake off the solvent on $80^{\circ} \mathrm{C}$ water bath, set into refrigerator to freeze rapidly, and put into the dryer to dry for 24 hours after curing. Solid dispersion of draconis and $\mathrm{PVP}_{\mathrm{k} 30}$ were obtained at ratio of $1: 1,1: 2,1: 3,1: 4,1: 5,1: 6$, and it was used for a backup by a 60 mesh sieve filtration after grinding. The sanguis draconis-PEG-6000 solid dispersion was prepared by melting method. Sanguis draconis and PEG-6000 (Shanghai Chemicals Supply Station) were mixed by the mass ratio after 60 mesh sieve powder filtration. The carrier was set on $80 \mathrm{oC}$ water bath until completely melted. Powder of sanguis draconis were added into solution, which were stirred vigorously and set into quickly the refrigerator to freeze. Solid dispersion of draconis and PEG-6000 were obtained at ratio of $1: 1,1: 2,1: 3,1: 4,1: 5,1: 6$, and it was use for a backup by a 60 mesh sieve filtration after grinding.

Detection wavelength HP-8452A UV spectrophotometer (Hewlett-Packard Company) was used in the research. Draconis was dissolved with a small amount of ethanol. The solution of sanguis draconis and $\mathrm{PVP}_{\mathrm{k} 30}$, PEG-6000 were prepared with a appropriate concentration respectively by water. According to spectrophotometer, maximum absorption of sanguis draconis is at $280 \mathrm{~nm}$, scanning in the wavelength range of 200-400nm. It is flavonoids of draconis. $\mathrm{PVP}_{\mathrm{k} 30}$ and PEG-6000 have no interference with the absorption at $280 \mathrm{~nm}$ wavelength, so the content of sanguis draconis in solid dispersions was determined by UV spectrophotometric determination.

Preparation of standard curve Draconis was weighed precisely $20 \mathrm{mg}$, set into $50 \mathrm{ml}$ flask, and dissolved in ethanol of $10 \mathrm{ml}$. Then it was diluted with water to the mark and was shaken well for the stock solution. Stock solution of 0.5, 1.0, 2.0, 3.0, 4.0, $5.0 \mathrm{ml}$ were taken precisely into $50 \mathrm{ml}$ flask respectively, measured absorbance at $280 \mathrm{~nm}$ by water volume.

Determination of the recovery Three parts of stock solution of sanguis draconis were taken at 0.5 , 1.0, $2.0 \mathrm{ml}$ into $50 \mathrm{ml}$ flask. Those were added into PVP $\mathrm{k}_{\mathrm{k} 30}$ and PEG-6000 at ratio of 1: 4 by water volume. The content was determined by standard curve method, and the recovery rate was calculated. The results are shown in Table 1.

\begin{tabular}{lccccc}
\hline carrier & $\begin{array}{c}\text { High } \\
\text { concentration }\end{array}$ & $\begin{array}{c}\text { Medium } \\
\text { concentration }\end{array}$ & $\begin{array}{c}\text { Low } \\
\text { concentration }\end{array}$ & $\begin{array}{c}\text { The average } \\
\text { recovery }(\%)\end{array}$ & RSD\% \\
\hline PEG-6000 & 101.15 & 99.78 & 101.23 & 100.72 & 0.81 \\
PVP $_{\text {K30 }}$ & 98.7 & 99.3 & 100.5 & 99.5 & 0.92 \\
\hline
\end{tabular}

Table 1 . The result of recovery rate of different dispersion at three concentration $(n=3)$

Precision test Three concentration of the solution of the above two solid dispersion were measured respectively and repeated 5 times. Results of PEG-6000 and PVP $\mathrm{K}_{30} \mathrm{RSD}$ were $0.41 \%, 0.57 \%, 0.48 \%$ and $0.69 \%, 0.73 \%, 0.65 \%$, which indicated a good precision.

Dissolution experiments Smart dissolution apparatus (Tianjin University Radio Factory) was used in the research. Methods: slurry method; Rotating speed: $100 \mathrm{r} / \mathrm{min}$; Temperature: $37^{\circ} \mathrm{C}$; Medium: 900 $\mathrm{ml}$ distilled water; Determine: solids dispersion stored in drier were taken to grind before a 60 mesh 
sieve filtration. Powder of sanguis draconis solid dispersion were accurately weighed $100 \mathrm{mg}$, and they were thrown uniformly into a stirred solution of the dissolution medium. Solution was drown $5 \mathrm{ml}$ by time positioning, and $5 \mathrm{ml}$ blank media was supplied at 5, 10, 15, 20, 30, 40, 50, 60, $90 \mathrm{~min}$. The sample was filtered by $0.8 \mu \mathrm{m}$ microporous membrane, and measured in absorbance at $280 \mathrm{~nm}$ by the blank of water.

Dissolutions of the solid dispersion powder in various proportions are shown in Fig. 1. They were much higher than the original powder medicament. The dissolution percentage of draconis and $\mathrm{PVP}_{\mathrm{k} 30}$ solid dispersion at the rate of $1: 1,1: 2,1: 3,1: 4,1: 5,1: 6$ were $4.4,11.14,29.1,168.2,183.0$, 217.1 times (5 $\mathrm{min})$; 4.8, 18.4, 31.8, 119.5, 130.1, 140.3 times (10 $\mathrm{min})$; 6.5, 14.0, 55.1, 66.6, 71.7, 75.1 times (30 $\mathrm{min})$; and 5.1, 11.8, 49.8, 53.8, 59.3, 59.9 times (60 $\mathrm{min}$ ) as much as drugs original powder. Dissolution order of solid dispersions of sanguis draconis with $\mathrm{PVP}_{\mathrm{k} 30}$ in different proportions was $1: 1<1: 2<1: 3<1: 4<1: 5<1: 6$. With the increase of the amount of carrier, the rate of drug dissolution increased. Compared the percentage of drug release at $30 \mathrm{~min}$ and 60 min by twenty-two multiple analysis of variance, results showed that solid dispersion at the rate of 1: 1, 1:2, 1: 3, 1: 4, 1: 5, 1: 6 had a big difference from raw powder in dissolution rate, having a significant differences between the groups $(\mathrm{P}<0.05)$.

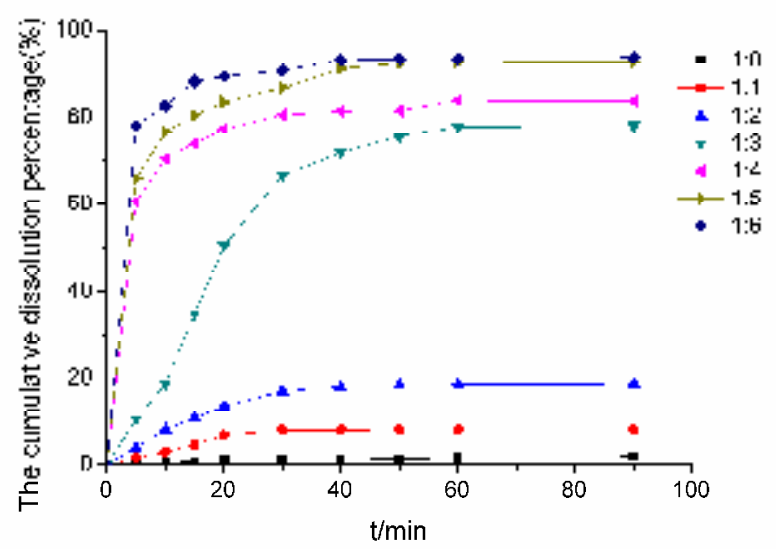

a

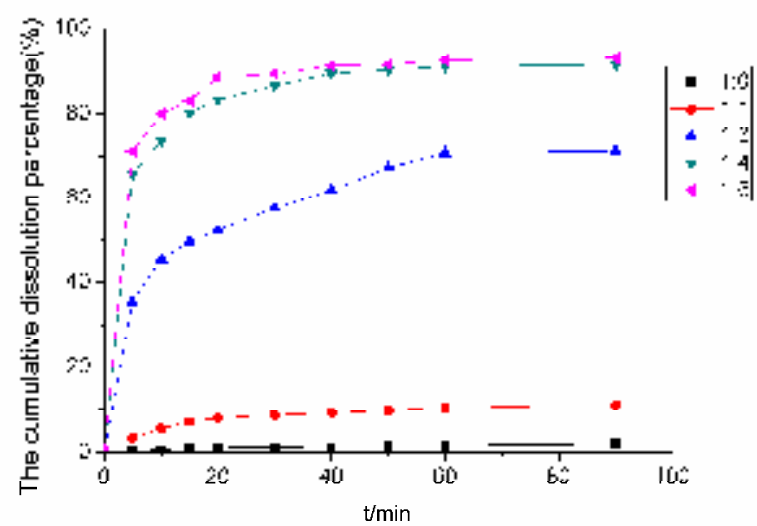

b

Fig. 1 In vitro dissolution rate of Sanguis draconis with $\mathrm{PVP}_{\mathrm{k} 30}$ solid dispersion (a) and PEG-6000 solid dispersion (b)

The dissolution percentage of draconis and PEG-6000 solid dispersion at ratio of 1:1, 1:3, 1:4, and 1:6 were $9.2,98.5,182.8$, and 197.5 times ( $5 \mathrm{~min}$ ); 9.4, 77.2, 124.7, and 135.5 times (10 $\mathrm{min})$; 7.2, $42.9,71.5$, and 73.8 times(30 $\mathrm{min})$; and 6.7, 45.3, 58.4, 59.3 times (60 $\mathrm{min}$ ) as much as drugs original powder. Dissolution order of solid dispersions of sanguis draconis with PEG-6000 in different proportions was 1: $1<1: 3<1: 4<1: 6$. With the increase of the amount of carrier, the drug dissolution rate increased. The percentage of drug release was compared at $30 \mathrm{~min}$ and $60 \mathrm{~min}$ by twenty-two multiple analysis of variance. Results showed that solid dispersion of 1:3,1:4,1: 5,1: 6 had a big difference from drug raw powder in dissolution rate, having a significant differences between the groups $(\mathrm{P}<0.05)$. This can be more rational and effective to use resources. Solid dispersion technology is one of the effective methods of improving insoluble drugs in vitro dissolution rate. $\mathrm{PVP}_{\mathrm{k} 30}$ and PEG-6000 are commonly used for carriers to prepare solid dispersions.

\section{Conclusions}

Draconis-PVP $\mathrm{k}_{\mathrm{k} 30}$ and draconis-PEG-6000 solid dispersion were prepared by sanguis draconis, and the dissolution rates of different samples in vitro were compared. We could find that draconis was insoluble in water from the dissolution profile, and various proportions of two carrier solid dispersion could significantly improve the solubility and dissolution rate of draconis. Experimental results showed that the dissolution rate of the drug had significant differences between the various 
proportions of solid dispersions of the original powder. The dissolution rates accelerated significantly with the increase of the proportion of the carrier. When the ratio of drug and carrier were reached at $1: 4$, dissolution of solid dispersions of two carriers were over $70 \%$ at $10 \mathrm{~min}$. The effect of solubilization was very obvious. Dracorhodin has the thermal instability in draconis [7]. When solid dispersions are prepared, we should pay attention to the temperature of water bath under $80{ }^{\circ} \mathrm{C}$ for ensuring the effectiveness of drugs. In the experiment, we found that $\mathrm{PVP}_{\mathrm{k} 30}$ as a carrier was better than PEG-6000 in dissolution. Therefore, $\mathrm{PVP}_{\mathrm{k} 30}$ as carrier of sanguis draconis solid dispersion is better.

\section{References}

[1] National Pharmacopoeia Committee: Chinese Pharmacopoeia, part I, (Chemical Industry Press, Beijing 2005).

[2] M.H. Fu, J. Fang, Z.J. Wang, L.Y. Tang, H.J. Yang, W. Li, X.Y. Yang, L.Q. Huang, Pharmacological research and clinical application of sanguis draconis, Lishizhen medicine and material medica research. Vol. 21 (2010), p. 1498.

[3] R.X. Yan: Water soluble polymer, (Chemical Industry Press, Beijing 1998).

[4] S.M. Liu, S. Zhang, Q.Y. Yao, Research on preparation and dissolution rate of nifedipine solid dispersions, Journal of Guangdong Pharmaceutical University. Vol. 23 (2007), p. 148.

[5] L.Y. Ding, P.F. Xia, C.Q. Yang, Preparation and evaluation of sustained release microsphere of Sanguis Draconis in vitro, China Journal of Chinese Materia Medica. Vol. 32 (2007), p. 388.

[6] Y.H. Sui, L.Q. Si, G. Li, Study on Preparation and Dissolution of Ciclosporin Solid Disperser in vitro, China Pharmacist. Vol. 7 (2004), p. 918.

[7] S.Q. Xu, Y. Hao, Q. Zhao, Stability of Chinese medicine of Sanguis Draconis, Chinese Traditional Patent Medicine. Vol. 28 (2006), p. 586. 